\title{
Multifluorescence High-Resolution Episcopic Microscopy for 3D Imaging of Adult Murine Organs
}

\author{
Claire Walsh,* Natalie A. Holroyd, Eoin Finnerty, Sean G. Ryan, Paul W. Sweeney, \\ Rebecca J. Shipley, and Simon Walker-Samuel
}

3D microscopy of large biological samples $\left(>0.5 \mathrm{~cm}^{3}\right)$ is transforming biological research. Many existing techniques require trade-offs between image resolution, sample size, and method complexity. A simple robust instrument with the potential to conduct large-volume 3D imaging currently exists in the form of the optical high-resolution episcopic microscopy (HREM). However, the development of the instrument to date is limited to single-fluorescent wavelength imaging with nonspecific eosin staining. Herein, developments to realize the potential of the HREM to become multifluorescent high-resolution episcopic microscopy (MF-HREM) are presented. MF-HREM is a serial-sectioning and block-facing wide-field fluorescence imaging technique, which does not require tissue clearing or optical sectioning. Multiple developments are detailed in sample preparation and image postprocessing to enable multiple specific stains in large samples and show how these enable segmentation and quantification of the data. The application of MF-HREM is demonstrated in a variety of biological contexts: 3D imaging of whole tumor vascular networks and tumor cell invasion in xenograft tumors up to $7.5 \mathrm{~mm}^{3}$ at resolutions of $2.75 \mu \mathrm{m}$, quantification of glomeruli volume in the adult mouse kidney, and quantification of vascular networks and white-matter track orientation in adult mouse brain.

optically transparent through delipidation and refractive index matching, ${ }^{[1-3]}$ thus enabling visible light to penetrate large (several centimeters) samples fully. Serial-sectioning physically cuts the sample, exposing the deeper tissue layers and removing the need for visible light to penetrate the tissue. Cleared samples can be imaged using techniques such as lightsheet microscopy ${ }^{[2,4]}$ and optical projection tomography (OPT). ${ }^{[3,5]}$ While clearing has been successfully applied to many organs and tissues, the plethora of different protocols can be daunting for new users and a researcher must carefully consider the trade-off associated with each method, e.g., effectiveness of tissue clearing (particularly for large organs) verses stain preservation (endogenous fluorescence and lipophilic dyes), morphological changes, time, and complexity. ${ }^{[6-8]}$

In serial-sectioning, samples are embedded in a hard supporting material, such as resin or paraffin, forming a block. Serial sections can then either be cut, mounted,

\section{Introduction}

3D optical imaging for large $\left(>0.5 \mathrm{~cm}^{3}\right)$ intact samples is an increasingly utilized tool in many areas of biomedical research, driven by the desire to understand the $3 \mathrm{D}$ structure of biological systems across multiple biological length scales. All 3D optical microscopy techniques aiming to image large samples must overcome the opacity of the tissue to visible light caused by scatter and absorption; tissue clearing and serial-sectioning are two of the most common approaches. Tissue clearing renders tissue

Dr. C. Walsh, N. A. Holroyd, Dr. E. Finnerty, Prof. S. Walker-Samuel Centre for Advanced Biomedical Imaging

University College London

London WC1E 6DD, UK

E-mail:c.walsh.11@ucl.ac.uk

The ORCID identification number(s) for the author(s) of this article can be found under https://doi.org/10.1002/adpr.202100110.

(C) 2021 The Authors. Advanced Photonics Research published by Wiley$\mathrm{VCH} \mathrm{GmbH}$. This is an open access article under the terms of the Creative Commons Attribution License, which permits use, distribution and reproduction in any medium, provided the original work is properly cited.

DOI: 10.1002/adpr.202100110 stained, and individually imaged, or imaging and sectioning of the block face can be interleaved (i.e., an image of the block face taken after each successive section is cut). For the former approach, the 3D alignment of the images is a nontrivial challenge due to the significant distortions and misalignments that occur during sectioning and subsequent processing. ${ }^{[9]}$ In contrast, the second approach, serial-section block-face (SSBF) imaging, produces inherently aligned images, thereby overcoming the slice alignment challenge and preventing the potential loss of data through sections damaged during cutting.

Prof. S. G. Ryan

Department of Physics, Astronomy and Mathematics

University of Hertfordshire

College Lane, Hatfield AL10 9AB, UK

Dr. P. W. Sweeney

Cancer Research UK Cambridge Institute

Li Ka Shing Centre

University of Cambridge

Cambridge CB2 ORE, UK

Prof. R. J. Shipley

Department of Mechanical Engineering

University College London

London WC1E 6BT, UK 
However, SSBF requires whole-mount staining (similar to light-sheet imaging) and suffers a loss of axial resolution, due to contamination of the image plane by out-of-focus light from below the block's surface (subsurface fluorescence)..$^{[10,11]}$ The addition of optical-sectioning capabilities, such as two-photon and structured illumination to SSBF instruments, has largely overcome the subsurface fluorescence issue ${ }^{[12-15]}$ but at the cost of dramatically increasing such instruments' complexity and technical specification. These instruments are highly complex, predominantly custom built, and require extensive expertise to align and maintain. ${ }^{[2,15]}$ This puts SSBF imaging beyond the reach for the majority of biomedical researcher labs and thus creates a niche for a technically simpler SSBF imaging technique for cases where a clearing-based approach is impossible or undesirable. A commercially available SSBF system, the Optical HREM (Indigo Scientific), has been in existence for several years and robust protocols for its use are well established. ${ }^{[16]}$ The system (Figure 1A) comprises a compound microscope head
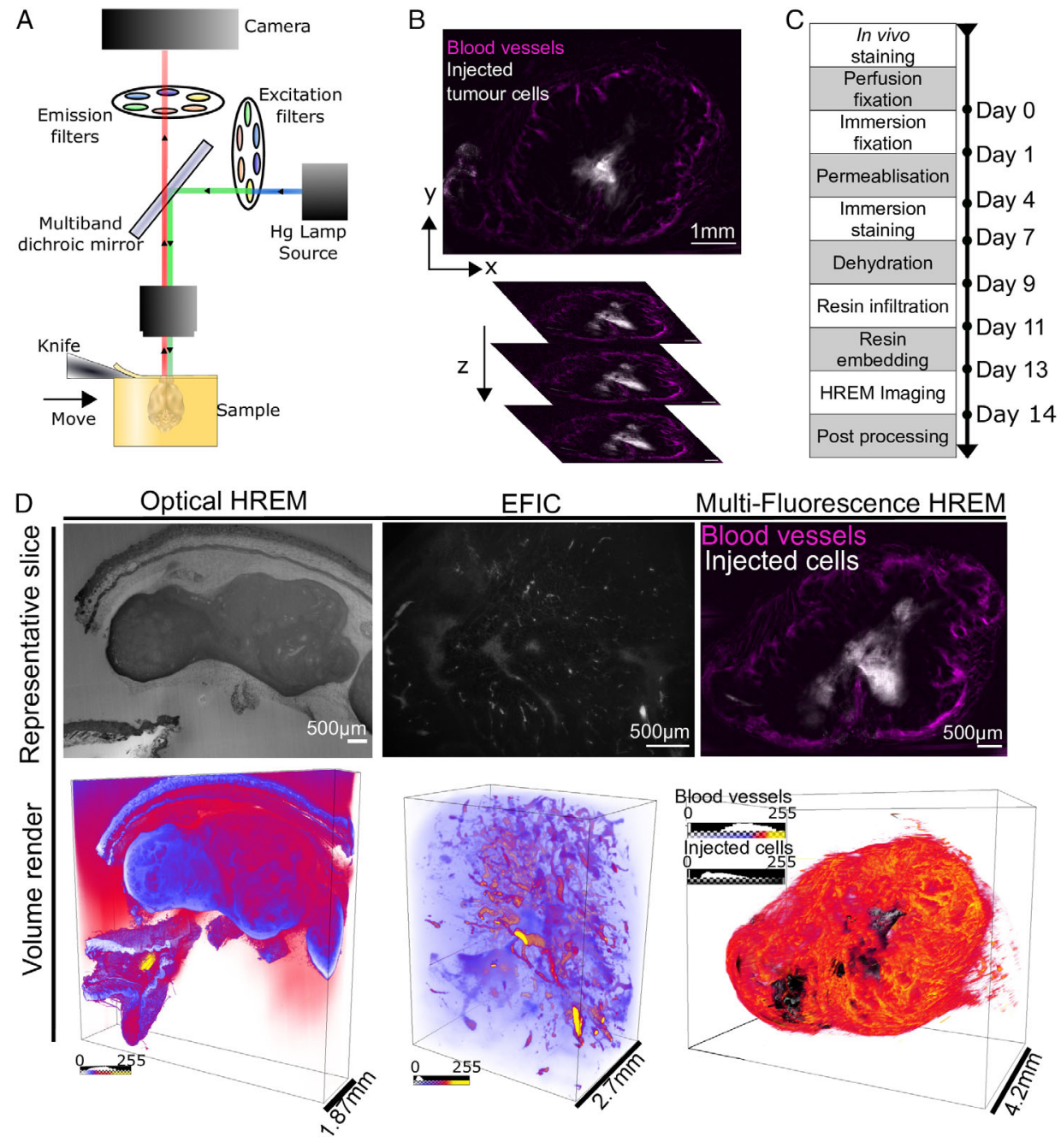

Multi-Fluorescence HREM
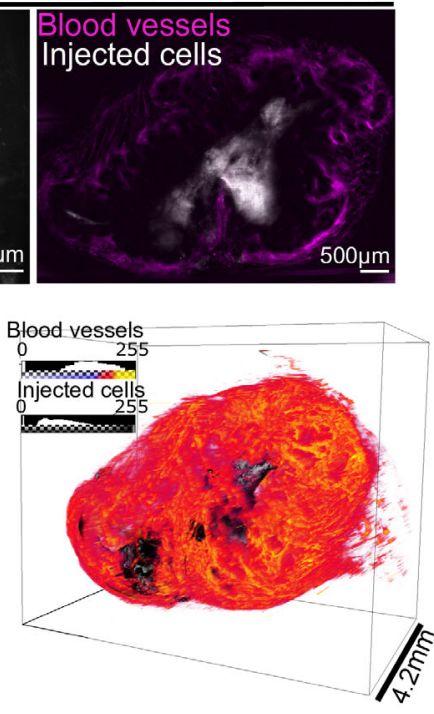

Figure 1. A) The HREM instrument consists of a fluorescent microscope with $1 \times$ objective lens (NA:0.25) and a variable zoom which provides fields of view ranging from $25 \mathrm{~mm}$ down to $2.3 \mathrm{~mm}$. Biological samples are held within a removable sample holder under the microscope objective on a z-translational stage to enable sections to be cut with a horizontally aligned, automated sectioning blade. Single-use tungsten carbide blades allow large samples to be cut. The sample is illuminated by a mercury vapor lamp, with separate excitation and emission filters for multiple-wavelength imaging. B) Single slices are inherently aligned leading to simple 3D volume rendering of xenograft tumor model. Vascular network as stained by i.v-administered lectin and injected cells (stained via the medium-term cell-tracing dye CM-Dil) can be clearly identified. C) The MF-HREM sample preparation, acquisition, and image postprocessing timeline for a typical multistained sample. For animal models, the sample is collected following perfusion fixation; in some cases, the sample is stained in vivo, prior to fixation. The sample is fixed overnight in paraformaldehyde (PFA) before being whole-mount stained (small mount stain or antibody). As almost all candidate resins are immiscible with water, samples must be dehydrated before polymerization. Once staining is complete, the sample is dehydrated using a series of organic solvents, followed by infiltration with a three-part glycol methanlacrylate acrylic (GMA) resin. Finally, the sample is set within the final resin block in the desired orientation and attached to a chuck for mounting to the instrument. Sample imaging with multiple wavelength channels is automated. D) A demonstration of the three HREM imaging methods, utilizing different sources of contrast, in xenograph tumor models. Optical HREM derives' contrast from positive, nonspecific eosin staining. Tissue autofluorescence provides contrast in EFIC. MF-HREM uses multichannel fluorescence labeling to visualize multiple specific cellular targets simultaneously. 
(Olympus MVX10) (as widely used in various light-sheet setups), ${ }^{[2]}$ a light source (LED or arc lamp) with a polychromatic mirror, and an excitation and emission filter wheel for wavelength selection. A charge-coupled device (CCD) camera (users' choice) records images after each section. The key feature of the system is the inbuilt automated microtome and software interface for alternate block-face fluorescent imaging and thin section cutting (down to $0.86 \mu \mathrm{m}$ ).

The Optical HREM instrument and methods were originally developed as a platform for phenotyping mouse models ${ }^{[17-22]}$ and have since been more widely applied. ${ }^{[23-25]}$ The main limitation in its use to date has been the absence of protocols and tools for using multiple targeted contrast agents. The vast majority of HREM to date has utilized a negative-contrast eosin approach (via the property of eosin, when bound to eosinophilic proteins, to inhibit the fluorescence of unbound eosin in the embedding resin). This produces images with an appearance similar to the inverse of traditional eosin staining in histology (Figure 1D). One exception has been the use of nonfluorescent resin with native autofluorescence. ${ }^{[26]}$ This autofluorescence approach (termed episcopic fluorescence image capture (EFIC)) was tested on mouse embryos but does not have potential to target specific structures or multiplex stains (Figure 1D). Moreover, the resolution using autofluorescence is far coarser than the eosin staining approach, as no postprocessing solutions to recover the axial resolution have been developed. ${ }^{[26]}$

Seeing the potential for this instrument in the 3D imaging field, we have developed the necessary sample preparation techniques and an axial resolution postprocessing method to enable dual fluorescent labeling, multichannel imaging, and quantification of specific biological structures in tissue samples $>0.5 \mathrm{um}^{3}$, at resolution up to $2.75 \mu \mathrm{m}$ using the Optical HREM instrument (Figure 1B). In this article, we present the optimization of the MF-HREM methodology, including stain penetration and resin embedding. We also describe a two-stage approach to recovering axial resolution, first using an opacifying agent (orasol black [OB]) to limit light transmission into the sample and second using deconvolution in postprocessing with a point-spreadfunction (PSF) estimated from the image stack itself. Finally, we demonstrate the wide applicability of MF-HREM by: 1) quantifying glomeruli volume in adult mouse kidneys; 2) segmenting vascular networks and invasive cells in a mouse tumor xenograft model; and 3) segmenting vascular networks and quantifying white-matter tract orientation in a mouse brain. We show here that these developments greatly broaden the potential applications of HREM and provide a large-volume 3D imaging platform that is accessible to a wide range of researchers.

\section{Results}

\subsection{Optimization of Sample Preparation}

The pipeline for MF-HREM is straightforward and does not require specialist equipment (Figure 1C). Each stage of the pipeline requires optimization for a specific experiment (organ and stain combination) and we have conducted these optimizations for a variety of adult mouse organs and experimental conditions. These optimized protocols can serve as a starting point for other experimental conditions and demonstrate the breadth of potential applications for MF-HREM.

\subsubsection{Staining}

As MF-HREM requires whole-mount staining prior to dehydration and resin infiltration, stain compatibility with the process must be established. We have tested a wide variety of stains and assessed their compatibility with various dehydrants and resins. We have particularly focused on providing robust counterstains for cell nucleus (HCS Nuclear Mask [NM]), cytoplasm (HCS Cell Mask [CM]) and cells membrane (Wheat Germ Agglutinin), as well as for vascular-staining lectin-dyelight conjugate (a full list of stains can be found in Table S2, Supporting Information). It is noteworthy that the lipophilic stain, CMDiI, is compatible with MF-HREM where it is not with many clearing techniques. ${ }^{[7]}$

Whole-mount staining requires homogenous and rapid stain penetration, which can be improved by increasing tissue permeability. Four methods to increase the permeability of tissue samples that have been previously used ${ }^{[1,5,27,28]}$ were compared: freeze-thaw method, proteinase $\mathrm{K}(\mathrm{P}[\mathrm{K}])$ digestion, iDISCO (which combines several mild detergents), ${ }^{[1]}$ and saponin. The comparison of the four methods on adult mouse kidneys showed that saponin treatment significantly increased stain penetration, compared with the control case $(p=0.04)$ (paired $t$-test $N=4)$. The iDISCO method also increased stain homogeneity $(p=0.055)$ compared with control kidneys (Figure 2A-C).

Alternative staining routes, such as intravascular (i.v.) injection, are also compatible with MF-HREM in animal models. For vascular staining, use of i.v. injection of fluorescently conjugated lectins is effective with the MF-HREM pipeline across a range of organs, as shown in Figure 5-7.

\subsubsection{Resin Infiltration and Embedding}

After staining, samples must be dehydrated and embedded in resin to provide mechanical stability during sectioning. Various commercial resins are used in histology, however, as these resins are designed to be manually cut and subsequently stained; they are not optimized for automated, thin sectioning, fluorescence preservation, or, in many cases, large samples. ${ }^{[2]}$ We investigated the compatibility of five commercial resins, which covered the three broad chemical categories for hard resins: methacrylate resins (Technovit 7100, Technovit 8100, and Lowicryl HM20), epoxy resin (Spurr), and acrylic resins (LR White). ${ }^{[2-31]}$ Resins were assessed for the time taken to set (quantitative), their compatibility with an opacifying agent OB (binary) (see Section 2.2.1 for further details), for their cut quality (semiquantitative) and for hardness (quantitative).

Technovit 8100 provided the best combination of cut quality, hardness, time to set, and compatibility with OB (Figure S1 and Table S3, Supporting Information). The addition of OB to Technovit 8100 showed that resin hardness decreased with increasing concentrations of $\mathrm{OB}$ and that this could be counteracted by increasing the concentration of the resin's secondary catalyst (Figure S1, Supporting Information). 


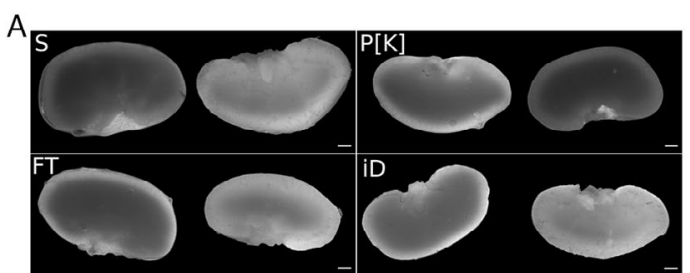

C
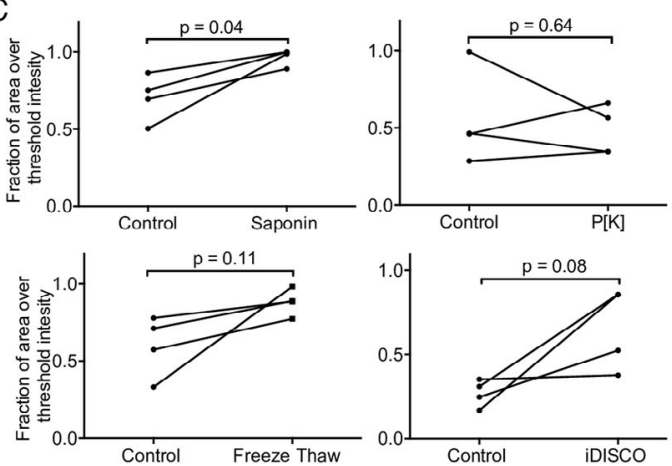

B
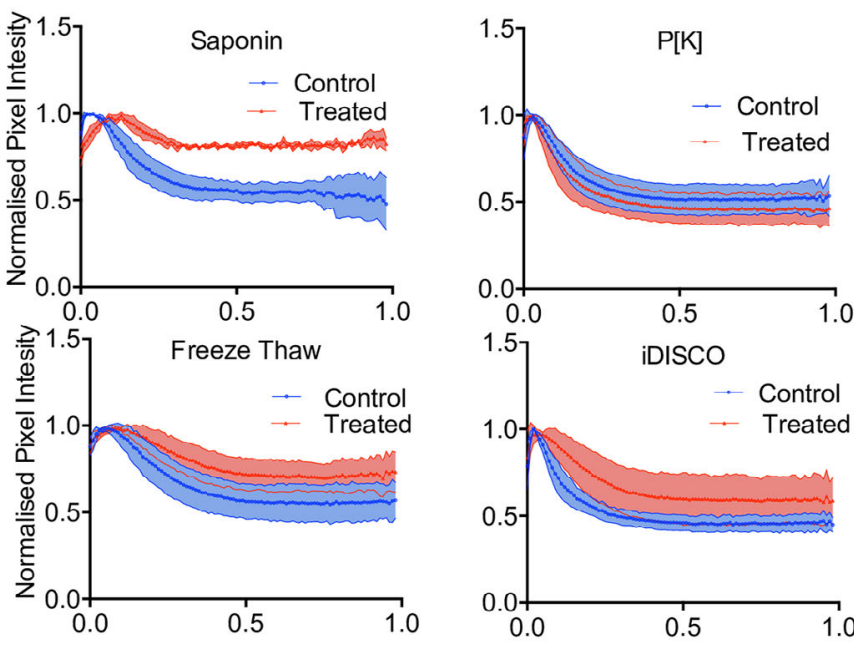

Normalised Distance from Kidney Edge

Figure 2. Optimization of stain penetration in adult mouse kidney samples. Four methods for improving stain penetration are compared: saponin treatment, proteinase $[\mathrm{K}]$ digestion, freeze-thaw, and iDISCO. For each method, four treated and four control kidneys were investigated, with one kidney from each animal used as a control for the contralateral kidney. A) Representative images of the kidneys, imaged as described in Section 5.2, alongside the control (contralateral kidney on the left and the treated kidney on the right) (scale bar: $1 \mathrm{~mm}$ ). B) The normalized MF-HREM signal intensity profile as a function of radial distance from the kidney edge. C) The fractional area of the kidney section image, above a threshold value (the same threshold was used for each treated kidney and matched control using default thresholding in Image|). Results of the paired t-test analysis demonstrate that saponin treatment significantly increased stain penetration $(p<0.05)$.

Finally, infiltration/embedding times for a variety of adult murine tissues were optimized to provide protocols for differently sized organs (Table 1).

\subsection{Minimizing Subsurface Fluorescence}

\subsubsection{Minimizing Subsurface Fluorescence with $O B$}

In any wide-field microscopy technique, fluorophores that are above or below the focal plane may be excited and their emission captured as out-of-focus light at the focal plane (Figure 3A). For MF-HREM and other SSBF techniques, this effect is asymmetric due to the physical sectioning of the sample above the focal plane. This leads to a characteristic comet tail-like artifact in the axial plane, which prevents image segmentation. To reduce this so-called subsurface fluorescence, opacifying agent (OB) may be added to the embedding resin. $\mathrm{OB}$ reduces the penetration of incident light into the block and absorb emitted photons from beneath the block's surface, thus reducing subsurface fluorescence. ${ }^{[32]}$

The extent to which OB reduces subsurface fluorescence is wavelength dependent. The transmission spectrum of $\mathrm{OB}$ is shown in Figure 3B. It has a broad absorption from the $450 \mathrm{~nm}$ to $650 \mathrm{~nm}$ range, with a steep increase in transmission in the near-infrared range $(>700 \mathrm{~nm})$. We have previously shown the decrease in subsurface fluorescence with increasing $O B$ concentration at wavelengths of $705 \mathrm{~nm} \cdot{ }^{[11]}$ When conducting MF-HREM, the wavelength-dependent absorption of OB as well as the type of structure and the staining intensity must be considered.

Table 1. Optimization of dehydration and embedding times for samples or various sizes.

\begin{tabular}{|c|c|c|c|c|}
\hline Reagent & $\begin{array}{l}>60 \mathrm{~mm}^{3} \text { Embryo (up to E12.5) } \\
\text { organoid or } 3 \mathrm{D} \text { culture }\end{array}$ & $\begin{array}{l}60-400 \mathrm{~mm}^{3} \text {, e.g., sciatic } \\
\text { nerve or embryo E14.5 to } \mathrm{Pl}\end{array}$ & $\begin{array}{l}0.4-1 \mathrm{~cm}^{3} \text {, e.g., heart, } \\
\text { kidney, brain, tumor }\end{array}$ & $>1 \mathrm{~cm}^{3}$ \\
\hline Acetone $50 \%$ & $1 \mathrm{~h}$ & $6 \mathrm{~h}$ & $12 \mathrm{~h}$ & $24 \mathrm{~h}$ (refresh at $12 \mathrm{~h}$ ) \\
\hline Acetone $70 \%$ & 1 & $6 \mathrm{~h}$ & $24 \mathrm{~h}$ (refresh at $12 \mathrm{~h}$ ) & $24 \mathrm{~h}$ (refresh at $12 \mathrm{~h}$ ) \\
\hline Acetone $80 \%$ & $1 \mathrm{~h}$ & $2 \mathrm{~h}$ & $2 \mathrm{~h}$ & $12 \mathrm{~h}$ (refresh at $6 \mathrm{~h}$ ) \\
\hline Acetone $100 \%$ & $15 \min \times 3$ & $1 \mathrm{~h} \times 3$ & $2 \mathrm{~h} \times 3$ & $3 \mathrm{~h} \times 3$ \\
\hline 50:50 Acetone: infiltration sol & $2 \mathrm{hrs}$ & $12 \mathrm{hrs}$ & $12 \mathrm{~h}$ & $24 \mathrm{~h}$ \\
\hline 25:75 Acetone: infiltration sol. & NA & $12 \mathrm{~h}$ & $12 \mathrm{~h}$ & $24 \mathrm{~h}$ \\
\hline $100 \%$ Infiltration sol. + vacuum & $2 \mathrm{~h}$ include $\mathrm{OB}$ & $12 \mathrm{~h}$ include $\mathrm{OB}$ & $\begin{array}{c}24 \mathrm{~h} \text { (refresh at } 12 \mathrm{~h} \text { ) include } \\
\text { OB after refresh }\end{array}$ & $\begin{array}{c}48 \mathrm{~h} \text { (refresh at } 24 \mathrm{~h} \text { ) include } \\
\text { OB at refresh }\end{array}$ \\
\hline
\end{tabular}



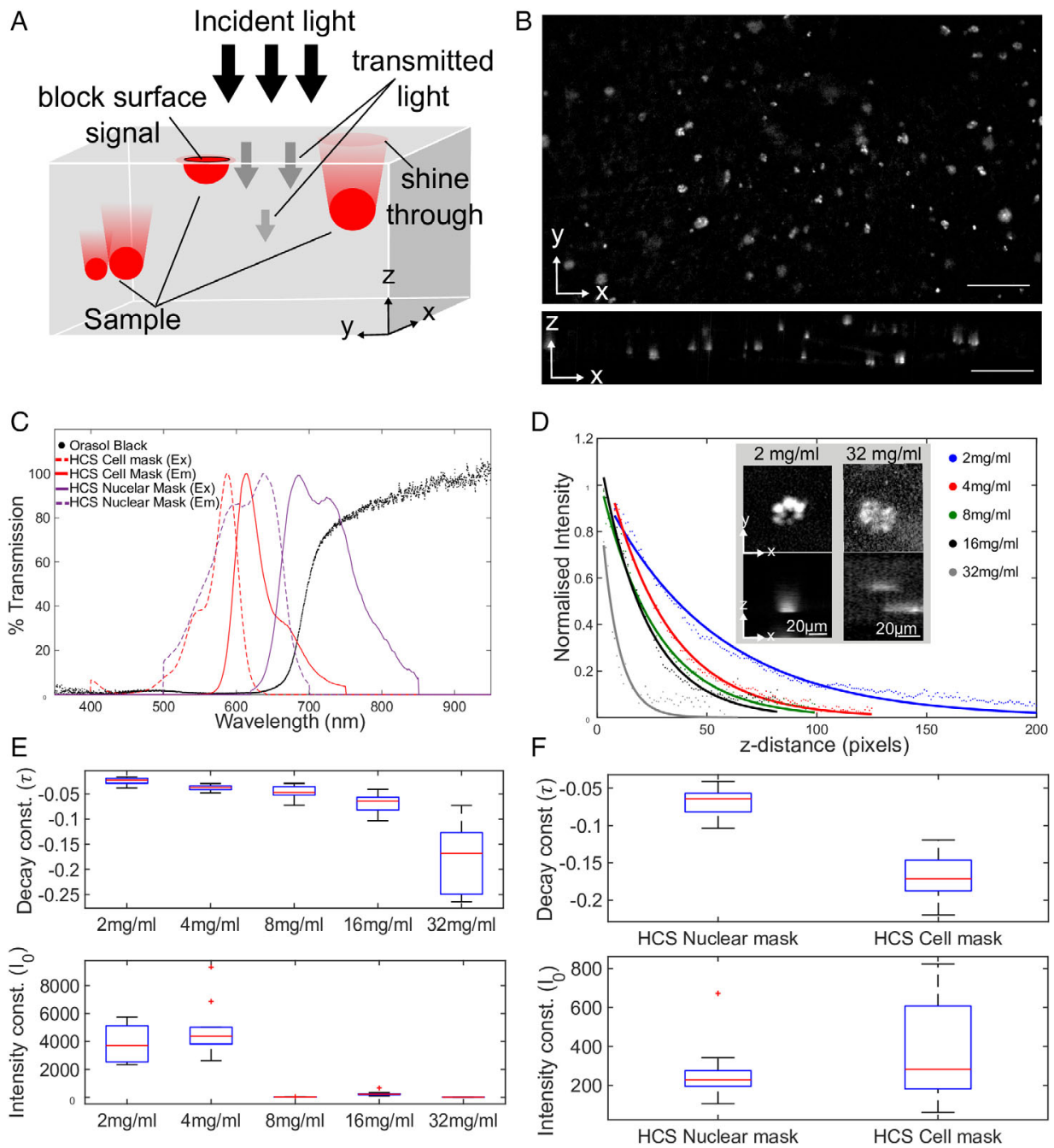

Figure 3. Characterization of $O B$ as an opacifying agent to reduce shine through with multiple fluorophores. A) Diagram demonstrating the origin of shine through. B) Graph showing the measured transmission spectrum of $\mathrm{OB}\left(0.1 \mathrm{mg} \mathrm{mL}^{-1}, 4 \mathrm{~mm}\right.$ path length), as well as two tested commercial stains HCS NM and HCS CM (spectrum from manufacturer) (adapted from the study by Walsh et al. ${ }^{[1]}$ ). OB has low transmission in the $400-625 \mathrm{~nm}$ range, which increases steeply in the 625-700 nm range. The CM spectrum falls almost entirely within the low-transmission band of OB, whereas the emission of NM falls in the section of steep increase in transmission. C,D) Representative images of cells in an in vitro 3D culture stained with NM (C) and CM (D) with $16 \mathrm{mg} \mathrm{ml}^{-1}$ of OB. The comet tail artifact can be seen in the $x z$ plane image particularly for NM, (scale bar $\left.=200 \mu \mathrm{m}\right)$. E) Line profiles from C and D. The longer comet tail in the case of NM can be seen more clearly here, as can the lower signal-to-noise ratio for NM. F) A single-exponential fit to the mean intensity profiles for ten region of interest (ROIs) ( $x z$ plane) taken of single cells in the cultures stained with NM and CM with $16 \mathrm{mg} / \mathrm{ml}$ of OB (inset shows the same data normalized to initial intensity). E) Boxplots for the fit constants of the ten ROIs for CM and NM: exponential decay constant ( $\lambda$ ), initial intensity $\left(I_{0}\right)$, background $(c)$, and $R^{2}$ values. As expected from the transmission spectrum, $\mathrm{NM}$ has a significantly lower decay constant $(p=0.025)$. In addition, the lower signal-to-noise ratio seen in $(\mathrm{E})$ is quantified by the lower the $I_{0}$ of NM compared with $\mathrm{CM}\left(p=2 \times 10^{-5}\right)$ and the lower $R^{2}$ values $(p=0.035)$.

Figure 3B shows the transmission spectrum of two commercial counterstains HCS CM (a cytoplasm stain) and HCS NM (a nuclear stain). The CM spectrum falls almost entirely within the low-transmission band of $\mathrm{OB}$, whereas the emission of NM falls in the section of steep increase in transmission. Representative images in Figure 3C,D and the intensity profile plots in Figure 3E show the more pronounced comet tail artifact in the NM case than the CM case for the same concentration of $\mathrm{OB}\left(16 \mathrm{mg} \mathrm{mL}^{-1}\right)$. Figure $3 \mathrm{~F}, \mathrm{G}$ quantifies this difference, showing that the exponential decrease in intensity through the $z$ plane has a significantly lower decay constant for
NM than CM. Initial intensity $I_{0}$ and $R_{2}$ values are also significantly lower for NM than CM (Figure 3G), indicating the reduced signal-to-noise ratio for NM at this high OB concentration $\left(16 \mathrm{mg} \mathrm{mL}^{-1}\right)$. While these data demonstrate that a greater reduction in subsurface fluorescence can be achieved when using fluorophores fully within the absorption spectrum of $\mathrm{OB}$, other considerations must be factored in: the concentration of $\mathrm{OB}$ will also reduce the signal-to-noise ratio to different degrees, depending on the intensity of the stain used and the structure stained, and at high concentrations, OB interferes with resin setting. When conducting MF-HREM, 
the choice of OB concentration used must balance the requirements of minimizing subsurface fluorescence, achieving adequate signal-to-noise for image processing, and optimum resin setting. The wavelength-dependent OB absorbtion, relative intensities of multiple stains, and image segmentation goals will all have to be considered. In the example applications we present here, OB concentrations between 1 and $4 \mathrm{mg} \mathrm{mL}^{-1}$ were found to be optimal depending on the structures imaged and fluorophores used (see Section 3 and Table S1, Supporting Information).

\subsubsection{Minimization of Shine-through Artifact with Image Post Processing}

As OB cannot be used to entirely eliminate subsurface fluorescence, we applied a postprocessing method developed to deconvolve signal from SSBF modalities and enable segmentation. ${ }^{[11]}$ Briefly, the method uses structures from within the image stack to estimate the parameters of a synthetic asymmetric PSF. This PSF is used to deconvolve the image, and a priori geometric constraints of the structures (e.g., the orthogonality of blood

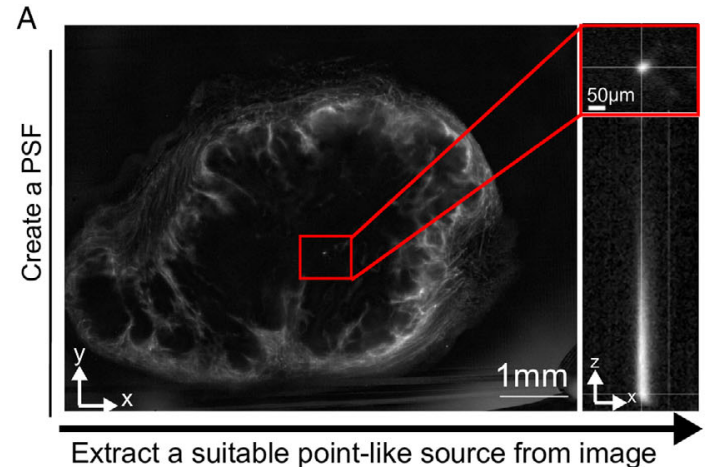

B

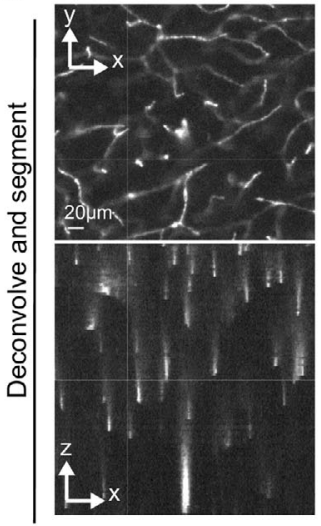

Pre-processed stack

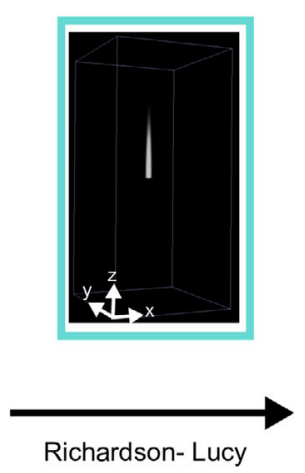

deconvolution

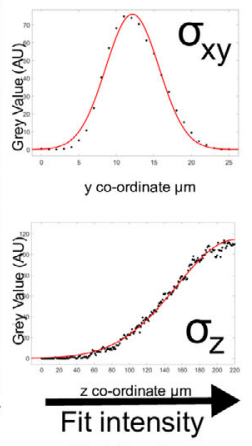

distribution

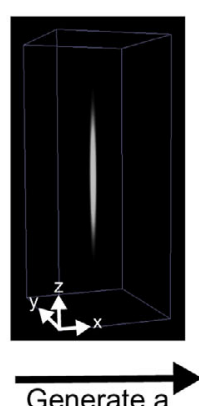

Generate a symmetric PSF

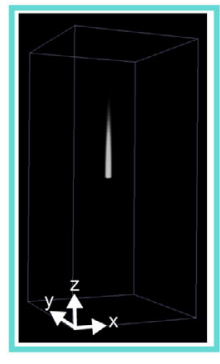

Zero lower half

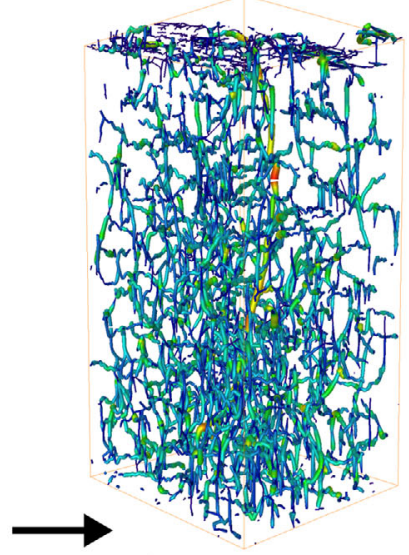

Segmented stack

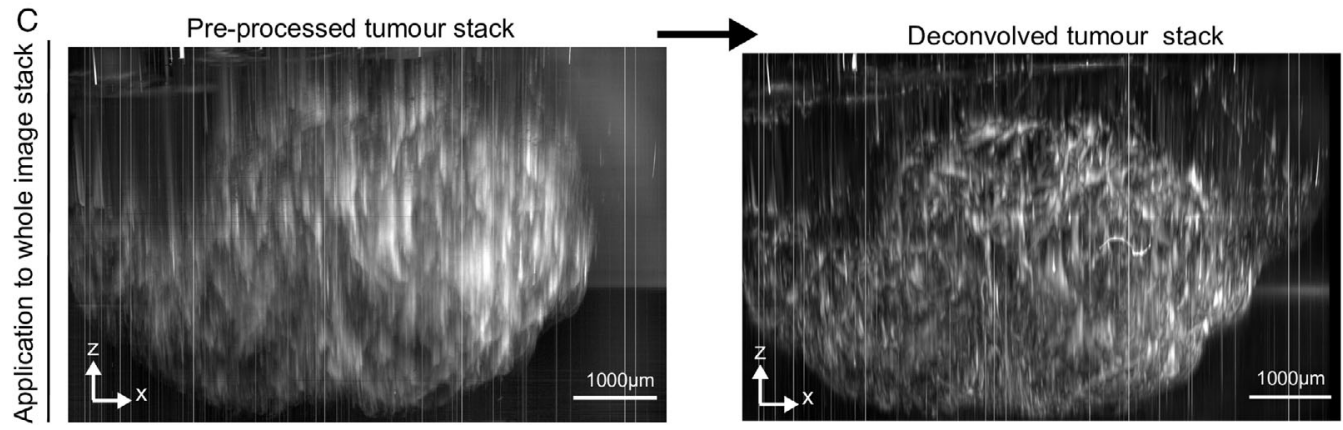

Figure 4. A) The pipeline for the extraction of a PSF from an image stack of a subcutaneous tumor with microvascular stained via injection of LectinDyelight649. Suitable point-like sources are found in the image stack, cropped from the image, and line profiles are used to fit $\sigma_{x y}$ and $\sigma_{z}$. A synthetic PSF is created with these parameters. ${ }^{[6]]}$ This PSF is then half zeroed to create the final PSF. B) Application of the PSF estimation and deconvolution method to a whole tumor vascular network improves axial resolution. C) Application of the PSF estimation and deconvolution method to a whole tumor cell distribution image improves axial resolution. 
vessels) are used to optimize the iteration number for a Richardson-Lucy (RL) deconvolution. ${ }^{[11]}$ Using a tilling approach with graphics processing unit (GPU) deconvolution enables fullsize MF-HREM images to be deconvolved efficiently and decreases the comet tail artifact to allow segmentation in many cases (Figure 4).

Measurements of the full width half maximum (FWHM) for small structures were used to parameterize a synthetic PSF model (Figure 4A).

A synthetic PSF was created using either the Gibson and Lanni Model or Gaussian model depending on pixel size. This model was parameterized from the mean measured FWHM (Gibson and Lanni) or standard deviation (Gaussian) of several selected small structures (Figure 4A) (as described in the study by Walsh et al. ${ }^{[11]}$ ). The lower half of the synthetic PSF was zeroed to replicate the asymmetry caused by serial-sectioning. This approach provides the practical advantages of using the image stack for PSF estimation, with the benefit of synthetic signalto-noise ratio. ${ }^{[33,34]}$ The asymmetric PSF was then incorporated into the widely used Richardson-Lucy deconvolution algorithm, an iterative method implemented in many open-source software repositories. ${ }^{[10,35]}$ This approach was applied to image stacks of different magnifications, wavelengths, and concentrations of OB (see Table S1, Supporting Information, for imaging parameter values). In all cases there is improvement in axial resolution and in the case of higher OB concentration and high magnification, the approach successfully enables segmentation of large vascular networks (Section 3.2). For lower concentration of OB and lower magnification (Figure 4B,C), our approach enables automated quantification of subsections of the image (Section 3.2) and improves the comet tails' artifact appearance.

\section{Applications}

\subsection{Glomeruli Number and Volume in Adult Mouse Kidney}

The nephron is the microscopic structural unit of the kidney, comprising two parts: the renal tubule and renal corpuscle. The renal corpuscle contains a cluster of capillaries known as a glomerulus. Nephron number has been used in both human and animal models as a biomarker of both renal and cardiovascular disease. ${ }^{[36]}$ However, the total nephron number and glomerulus size are challenging to estimate from 2D sectioning techniques. Figure 5 shows an adult mouse kidney stained with a vascular marker, prepared and imaged using our MF-HREM pipeline. Glomeruli were segmented in the final image stack. MF-HREM pixel size was $2.17 \mu \mathrm{m}$ lateral, or "in plane," and $2.58 \mu \mathrm{m}$ axial (voxel volume: $12.14 \mu^{3}$ ), enabling identification of glomeruli, which were found to have a minimum volume of $2.4 \times 10^{4} \mu \mathrm{m}^{3}$.

Our MF-HREM analysis measured 100 glomeruli per cubic millimeter of kidney volume, with median glomeruli size of $1.88 \times 10^{5} \mu^{3}$ and mean of $2.2 \times 10^{5} \mu^{3}$. The number of glomeruli per unit kidney volume is consistent with distributions for wild-type adult mice conducted with light sheet ${ }^{[37]}$ (100-140 glomeruli per $\mathrm{mm}^{3}$ of kidney) and also $\mathrm{MRI}^{[36]}$ (74-90 glomeruli $\mathrm{mm}^{3}$ of kidney). Our median size is also within the bounds of those measured by lightsheet, ${ }^{[37]}$ stereology, and
MRI. ${ }^{[36]}$ This demonstrates the ability of MF-HREM to provide accurate quantitative information on organotypic functional units in adult mouse organs.

\subsection{Imaging Tumor Blood Vessels and Cell Invasion with MF-HREM}

Widely reported hallmarks of cancer include deregulation of angiogenesis and active invasion/metastasis. ${ }^{[38]}$ Angiogenic disruption is evident in solid tumors through highly complex and disordered blood vessel networks. These networks are responsible for the distribution of nutrients and drugs ${ }^{[3]}$ and are involved in bidirectional signaling that can stimulate cellular invasion and, ultimately, lead to metastasis. ${ }^{[39]}$ Multiplexed 3D imaging of whole blood vessel networks with tumor cell invasion can be used to interrogate the spatial relationship between deregulated angiogenesis and cell invasion.

Figure 6 shows MF-HREM images from a subcutaneous xenograft tumor mouse model initiated from the FaDu hypopharyngeal cancer cell line, where both tumor cells and the blood vessel network have been fluorescently labeled. Both stains are clearly visible throughout the image stack (Figure 6A), and 3D reconstruction of the entire dataset for both channels reveals the dense, branching vasculature at the periphery of the tumor, and the labeled cells primarily in the tumor center, which appeared to be nonperfused due to an absence of the vascular signal (Figure 6B). Single cells or cell clusters are also visible (yellow arrows in Figure 6A's inset) in a section of tumor separate from the main bulk. While it is unclear whether labeled cells are viable (which would require a different reporter strategy), these results demonstrate the ability of MF-HREM to quantify the 3D location of injected cells in tissue volumes of $\approx 1 \mathrm{~cm}^{3}$ several weeks after injection.

For the vascular imaging stain, Figure 6C,D shows a subsection of the tumor vasculature that has been segmented following image processing with our deconvolution approach. The chaotic nature of the vasculature is clear from both the appearance and the wide range of vessel sizes (Figure 6D). Such segmented vascular networks can be used in simulations of drug delivery ${ }^{[3]}$ and for investigating tumor vessel growth mechanism. ${ }^{[40]}$

\subsection{Imaging Brain Microvasculature and White-Matter Tracts in a Mouse Brain with MF-HREM}

Healthy brain development and function is dependent upon the intricate networks of both cerebrovascular and white-matter fibers. Changes in the structures of these networks are key indicators of a number of degenerative neurological diseases including Alzheimer's and Parkinson's. ${ }^{[4]}$

Visualizing these structures in 3D with MF-HREM can be used to improve our understanding of disease or potentially validate in vivo imaging tools such as MRI.

Figure 7 shows the application of MF-HREM in two instances: 1) where a brain is dual labeled with CMDiI as a white-matter marker and Lectin-Dyelight649 as a microvascular stain (Figure 7A-D) and 2) where a brain is dual labeled with CMDiI as a white-matter marker and HCS NM as a marker 

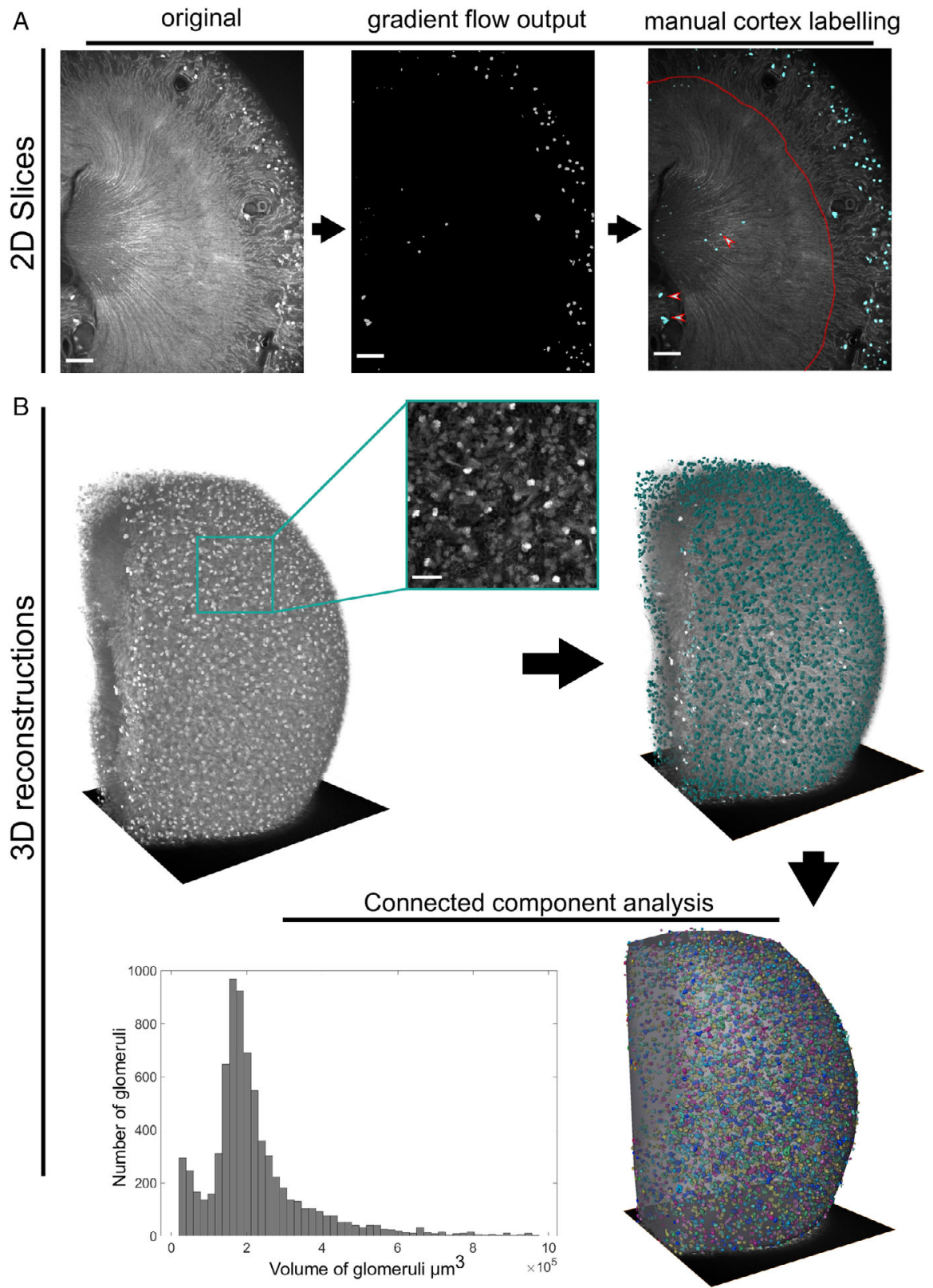

Figure 5. Quantification of glomeruli number from the Lectin-Dyelight649 channel of a multistained murine kidney. A) From left to right, 2D slices showing the unprocessed image, the segmented image produced by gradient vector flow algorithm, and finally, the manually corrected image after removal of structures that were segmented (white/red arrows) but fall outside the kidney cortex (red line). Scale bars: $500 \mu \mathrm{m}$ for all 2D slices. B) 3D view of image processing. The original image stack with inset showing the detail which can be seen on the kidney surface (scale bar: $200 \mu \mathrm{m}$ ); then, the segmentation via gradient flow algorithm in Vaa3 $\mathrm{D}^{[57-59]}$ and manual exclusion of points not in the cortex. The final step is the outcome of the connected components analysis and hence quantification of glomeruli number and volume distribution. These data show the expected distribution and size of glomeruli for healthy weight mouse as compared with other techniques. ${ }^{[36,37]}$

for cell distribution (Figure 7E,F). Figure 7B shows segmented vasculature from the high-resolution subvolume of the medulla. The observed microvasculature has a mean radius of $3.01 \pm 1.25 \mu \mathrm{m}$, with a large number of small vessels $(1.6-12 \mu \mathrm{m}$ diameter) oriented parallel the brain surface and a smaller number of larger (12-22 $\mu \mathrm{m}$ diameter) descending arterioles perpendicular to the brain surface (white arrows in Figure 7B's inset). This distribution is expected anatomically and accords well with those measured by other serial-sectioning modalities, e.g., MOST $(2.8-2.4 \mu \mathrm{m} \text { for matched subregions) })^{[41]}$ and for clearing techniques. ${ }^{[42]}$ It should be noted that no large vessels $(>22 \mu \mathrm{m})$ are visible in MF-HREM data, likely due to the preferential binding of lectin to microvasculature over larger vessels as noted previously. ${ }^{[43]}$

An additional feature of interest in brain microstructure is white-matter tract orientation. Previously, CMDiI has been used 

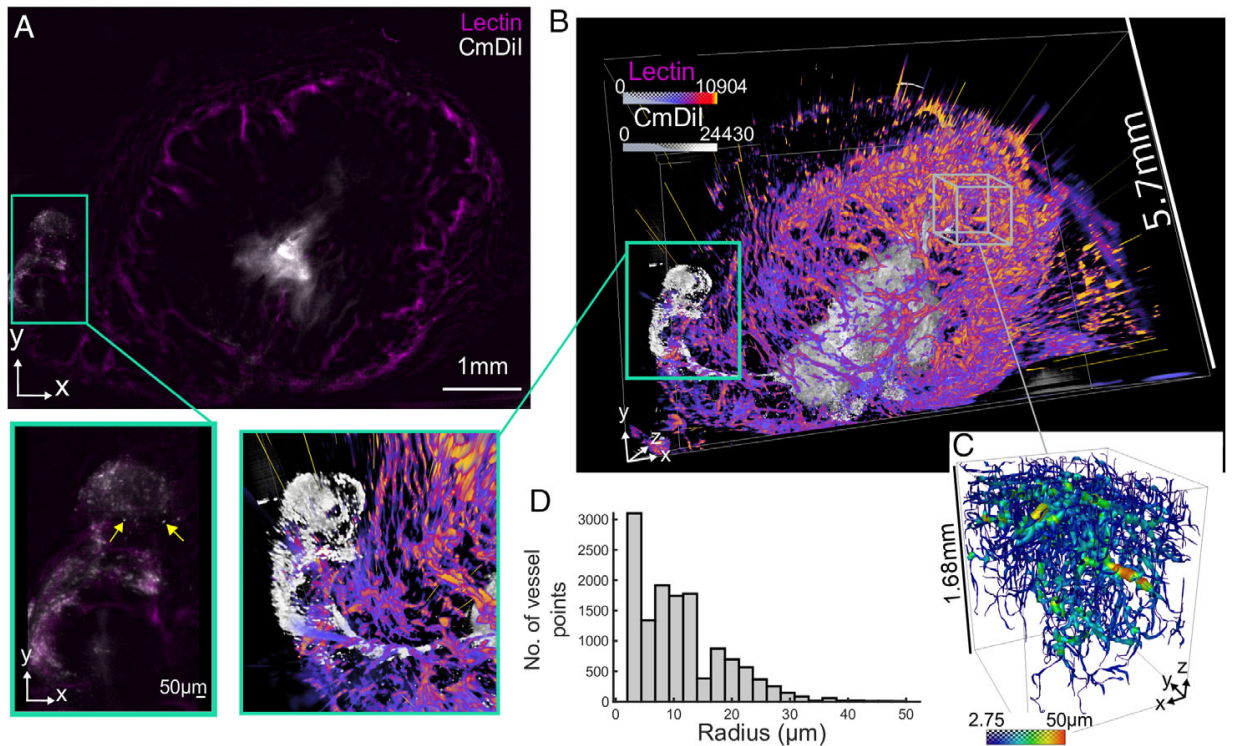

Figure 6. Xenograft tumor model analysis. A) A single representative slice with the two stains: CMDil for injected cell tracking (white) and Lectin-Dyelight 649 conjugate for microvascular staining (magenta). The inset shows a digitally zoomed-in portion of the image where small, approximately circular structures of cell size are indicated by yellow arrows. B) A 3D rendering of the two channels shows the tumor in its totality and the spatial arrangement of the injected cells within the vascular network. The highly perfused rim can be clearly seen. The inset shows the same digitally zoomed section as the 2D slice inset. C) Segmentation of a section of the vascular network following the PSF extraction described earlier. In this case the APP2 algorithm from the Vaa3D neuron-tracing plug-in set was used to segment and skeletonize the deconvolved image stack. $\left.{ }^{[57-60]} \mathrm{D}\right)$ The histogram of the vessel radii from $C$.

on histological sections of mouse brain to validate tractography from diffusion-weighted (DW)-MR in 2D. ${ }^{[44]}$ CMDiI effectively stains white-matter tracts due to its lipophilic nature and can be imaged with MF-HREM, allowing orientation analysis on the 3D volumes. Figure 7D,F shows white-matter orientation analysis for a small brain section and whole mouse brain, respectively. Orientation is quantified by the structure tensor of the image, which was estimated using the ImageJ plugin OrientationJ. ${ }^{[45-47]}$ The color bar shows the angle represented by the hue.

Cell distribution and density are also important markers of neurological development and pathology. Figure 7E shows a single slice at a higher magnification from the imaging volume in Figure 7F, and cell nuclei can be distinguished and demonstrate that nuclear staining has successfully been retained throughout processing. Further segmentation requires montaging of higherresolution images.

These data show that MF-HREM can be used in adult mouse brain as a tool for visualizing vascular networks, white-matter tracts, and cell distribution. Quantitative vessel analysis can be conducted on higher-resolution data and orientation analysis on whole brain images.

\section{Conclusion}

In this work, we have shown the development of sample preparation and image postprocessing in MF-HREM. We have demonstrated its applicability in adult mouse organs through a range of staining and quantification approaches.
Reducing the impact of subsurface fluorescence has been a key factor in realizing the potential of MF-HREM. Our current solution has combined two approaches: physically limit light transmission through the sample and deconvolution in postprocessing. Our deconvolution approach uses a sample-specific PSF, whilst circumventing the problem of poor signal-to-noise that would occur if the PSF is used directly from the image stack. Likewise, our optimization of tissue-specific staining and embedding protocols provides a solid foundation from which researchers hoping to apply this technique can build.

Further improvements to MF-HREM are possible in several areas of the imaging pipeline. The resin embedding and staining protocol can be improved by optimization to a specific biological problem. For example, a custom mould can be used for repeated preparation of similar samples. In addition, while dehydration and embedding times cannot be easily decreased without compromising the final imaging, automation of the process using an automated histological sample processor could improve consistency and enable faster protocol optimization for a specific application.

Diffusion staining alone will always struggle to achieve homogenous stain penetration in a timely manner. Even with increased stain penetration via saponin treatment, inhomogeneous staining is evident. This challenge is common to all whole-mount imaging techniques; thus, significant research effort continues to be directed at overcoming this, e.g., the use of nanobodies, genetically encoded reported, and perfusion staining. ${ }^{[48-50]}$

The two interdependent approaches used here to circumvent subsurface fluorescence could undergo more development to widen MF-HREM application. To further reduce light 


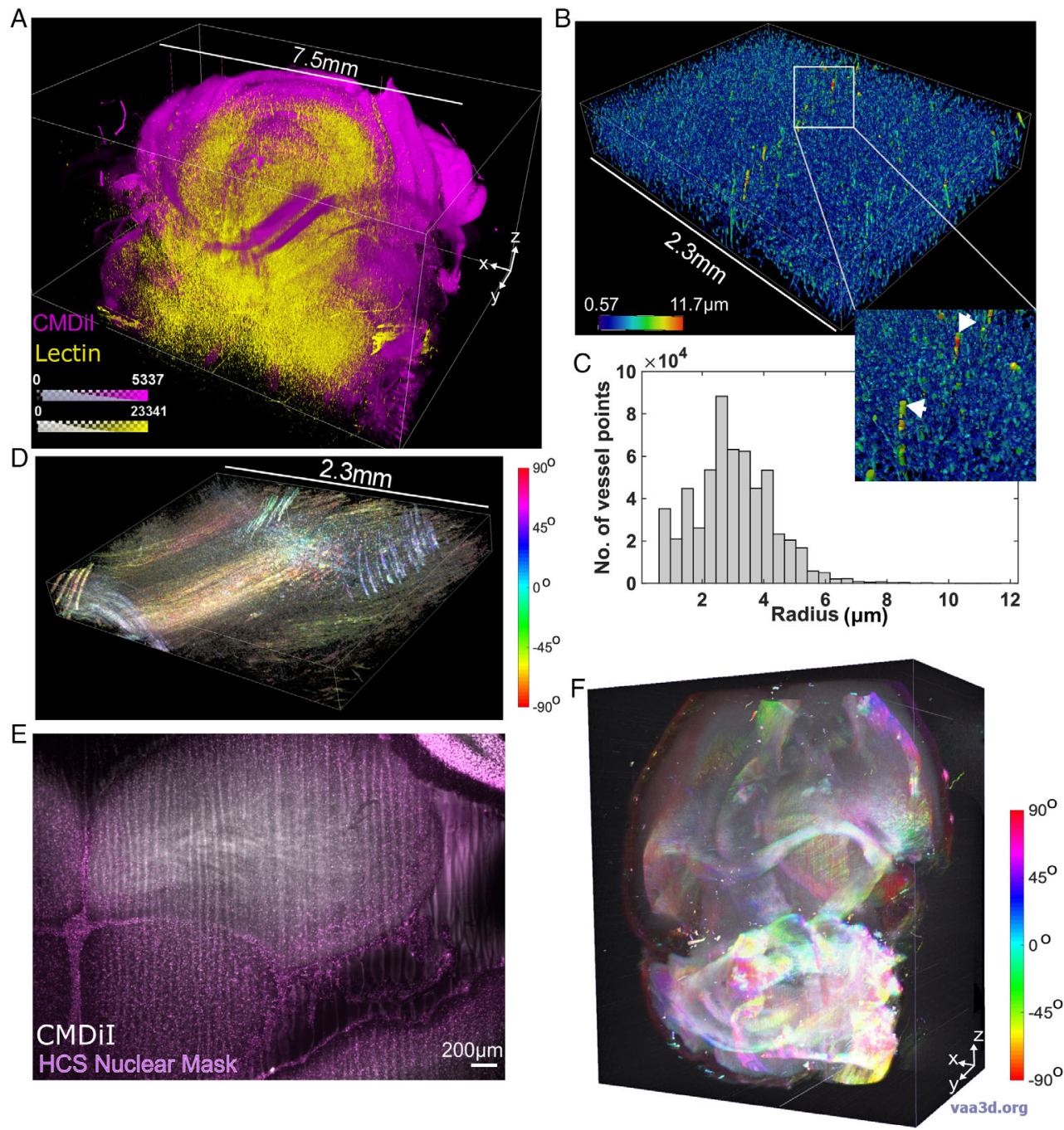

Figure 7. MF-HREM of two brain samples, one stained with CMDil Ex/Em 553/570 nm and Lectin-Dyelight 649 (Ex/Em 649/700) and a second stained with CMDil and HCS NM (Ex/Em 638/686). A) The volume overlay of both the CMDil (Magenta) and the Lectin (yellow). Color bars show pixel intensity. B) A high-resolution subvolume of the vasculature channel successfully segmented and skeletonized using the MF-HREM processing pipeline and the MOST tracing algorithm implemented in Vaa3D. C) The histogram of radii distributions. D) The same subvolume in the CMDil/white-matter channel; here, an orientation analysis has been conducted via calculation of the structure tensor of the image using a Gaussian gradient and eight-pixel window size. The hue, saturation, and brightness denote the orientation, coherence, and original image brightness, respectively. The color bar shows the angle represented by the hue. E) A polar histogram of (D), where all orientations with a coherence greater than a threshold value of 0.2 are displaced. It can be seen that there appears to be an even distribution of local orientations over this subvolume. F) Volume overlay of the CMDil channel (white) and the HCS NM channel (magenta) for a different brain sample. G) A single slice imaged at higher resolution in which nuclei can be clearly seen. $\mathrm{H}$ ) Orientation of white matter mapped over the whole brain. It was computed as in (D) but using a smaller window size of four pixels. The orientation can be seen to clearly follow the expected white-matter tracts.

transmission, alternative dyes could be incorporated into the resin or shorter-wavelength incident light used. ${ }^{[51]}$ Further improvements to the deconvolution could be made through more complex PSF models, for example, Gaussian mixture models $^{[52]}$ or spatially variant PSFs. ${ }^{[53]}$

Both optical clearing and serial-sectioning come with their own specific challenges. Approaches which keep the sample intact (thus requiring optical clearing) are limited in sample size and resolution by the working distance of the microscope objective lens ${ }^{[2]}$ and the need for broader light sheets to penetrate greater tissue depth. ${ }^{[12]}$ For applications where a serial-sectioning approach would be better suited, uptake of serial-sectioning techniques has been limited by the technicality of the custom-built instruments, ${ }^{[15,54]}$ leaving a niche for a robust, commercially available serial-sectioning technique.

In this work, we developed a pipeline for conducting MFHREM, which enables 3D, multiplexed fluorescence imaging of large tissue samples $\left(>0.5 \mathrm{~cm}^{3}\right)$, at high resolution. MFHREM is a block-facing technique, using a commercially available system (Optical HREM, Indigo Scientific, UK), that overcomes subsurface fluorescence, using a combination of an opacifying agent and image deconvolution. This technique 
can find wide applications through its avoidance of optical sectioning, tissue clearing, or the need for a custom-built instrument.

\section{Experimental Section}

Animal Models and Perfusion Fixation: All animal studies were licensed under the UK Home Office regulations and the Guidance for the Operation of Animals (Scientific Procedures) Act 1986 (Home Office, London, United Kingdom) and United Kingdom Co-ordinating Committee on Cancer Research Guidelines for the Welfare and Use of Animals in Cancer Research. ${ }^{[55]}$

For perfusion fixation, animals were euthanized via intraperitoneal (i.p.) injection of $100 \mathrm{mg} \mathrm{kg}^{-1}$ sodium pentobarbital (Animalcare, Pentoject) diluted in $0.1 \mathrm{ml}$ phosphate-buffered saline (PBS). Once anesthesia was confirmed, surgical procedures for cardiac perfusion were conducted for systemic clearance of blood. Heparinized saline $(20 \mathrm{~mL}$ with $1000 \mathrm{IU} \mathrm{mL}^{-1}$, maintained at $37^{\circ} \mathrm{C}$ ) was administered with a perfusion pump (Watson Marlow, 5058) at a flow rate of $3 \mathrm{~mL} \mathrm{~min}^{-1}$ to mimic normal blood flow. After the complete drainage of blood, mice were perfused with $20 \mathrm{ml}$ of $4 \%$ paraformaldehyde (PFA, VWR chemicals $4^{\circ} \mathrm{C}$ ). Organs were then removed and fixed for $12-24 \mathrm{~h}$ in $4 \%$ PFA at $4{ }^{\circ} \mathrm{C}$.

Stain Penetration: A total of 16 mice $(N=4)$ between 10 and 23 weeks old of various strains were used. Mice were perfuse fixed as described earlier and both kidneys were removed. Each animal was randomly assigned to one of the four groups (saponin, freeze-thaw, iDISCO and proteinase [K] digestion), and for each animal one kidney was randomly assigned to treatment and the contralateral kidney retained as a matched control. In the saponin, iDISCO, and proteinase $[\mathrm{K}]$ groups, the control kidney was maintained in PBS and at the same temperature as the treated kidney. For the freeze-thaw group, the control kidney was dehydrated and rehydrated through the same methanol series but with no freeze-thaw cycles applied. Details of the timing and solutions composition for each of the four groups are given in Supplementary Methods.

After treatment, all kidneys were stained for $94 \mathrm{~h}$ in $4 \mathrm{ml}$ of HCS NM (Thermo Fisher UK) at a concentration of $4 \mu \mathrm{L} \mathrm{mL}^{-1}$ in PBS at room temperature with constant agitation. They were then bisected at the urethra, and the cut surface was imaged on a glass-bottomed dish using the HREM microscope (all imaging parameters were kept constant).

Resin Testing: Multiple candidate resins were tested for setting time, compatibility with opacifying agents, final block hardness, and quality of cuts. Resins used were Technovit 7100 (Heraeus Kulzer, Germany), Technovit 8100 (Heraeus Kulzer, Germany), Spurr resin (Polysciences Inc, USA), LR White (Sigma-Aldrich, USA), and Lowicryl HM20 (Polysciences Inc, USA). Composition of each resin is given in Supplementary Methods.

Hardness testing was conducted using either a Shore durometer D or Shore durometer A on set blocks. Cut quality was assessed by imaging a block with a mouse kidney embedded within it on the Optical HREM (Indigo Scientific, UK) and quantifying the proportion of slices, which had areas of flaky resin or voids and the severity of resin imperfection. Waves vertical and horizontal were also incorporated into the cut quality assessment.

Spectroscopy: The transmission spectrum of OB was measured using an HG4000CG-UV-NIR (Ocean Optics) fiber-fed spectrometer. Samples of Technovit 8100 base sol. plus catalyst 1, with a low concentration $\left(0.1 \mathrm{mg} \mathrm{mL}^{-1}\right)$ of $\mathrm{OB}$, were measured in a polymethylmethacrylate (PMMA) semimicro cuvette over a $4 \mathrm{~mm}$ path length, from 350 to $95 \mathrm{~nm}$ with a QTH10/M (Thorlabs) continuum lamp. The transmission spectrum (Figure $3 \mathrm{C}$ ) was compensated for the cuvette reflectivity and PMMA absorption.

Subsurface Fluorescence Quantification: Standard 3D cell cultures were prepared as described (sup methods) and stained with HCS NM deep red or HSC NM. Samples were prepared for HREM, as shown in Table 1, with OB 45X (Stort Chemicals Ltd, Bishops Stortford, UK) mixed with Technovit 8100 at the last stage of resin infiltration (at concentrations of 2, 4, 8, 16, and $32 \mathrm{mg} \mathrm{mL}^{-1}$ ) prior to positioning and setting the sample within the final block.

Blocks were imaged over the full sample depth on the Optical HREM system using slice thickness $=1.72 \mu \mathrm{m} x y$, pixel size $=0.57 \mu \mathrm{m}$, gain $=9$ or 8 , and exposure $=1.0 \mathrm{~s}$ or $0.6 \mathrm{~s}$ for the $\mathrm{NM}$ and $\mathrm{CM}$ samples, respectively. Image stacks were downsampled in $x y$ to create isotropic voxels, Image stacks were resliced into $x z$ stacks (as shown in Figure 3C,D) Ten ROIs were manually drawn around isolated cells per sample. The ROIs were rectangular and fully enclosed all pixels with intensity above the background. The intensity profile of the ROI was plotted (pixel intensity vs $z$-direction). This signal was truncated at the maximum intensity and fit to a single-exponential model (Equation (1)) in Matlab using a nonlinear least squares approach with robust outlier elimination. Start values of 5000 for $I_{0}, 4000$ for $c$, and between 0 and 1 for $\lambda$ with limits $\pm \infty$ were used (other start values used made no difference to final fit parameters where the fit converged). The fits were used to quantify the change in subsurface fluorescence.

$I=I_{0} e^{-\lambda z}+c$

Image Postprocessing, PSF Fitting, and Deconvolution: HREM image stacks were inherently aligned and hence were reconstructed as a volume without any z-registration, alignment or overlap. Depending on the fluorophores used for labeling and the excitation emission filters used in the instrument, spectral unmixing (in the case of overlapping labeling spectra) or background subtraction to remove autofluorescence (in the case of shorter wavelength emission) may improve image appearance and aid in further segmentation (Figure S2, Supporting Information).

For deconvolution, the PSF parameters were estimated from the image stack as follows. The image stack was manually inspected for suitable small structures; these were cropped from the stack and a 3D median filtering with one pixel window size was applied to reduce noise. ${ }^{[56]}$ Line profiles through the small structures in $x, y$, and $z$ were fit either with a Gaussian (with offset in image) Fiji) or with an exponential decay from which the FWHM in $x y$ and $z$ were calculated (model depended on pixel size, see ST1 for details). A PSF was generated using with a Gaussian model or a Gibson and Lanni model. For the Gibson and Lanni model, the modeled wavelength and numerical aperture (NA) were iterated to minimize the difference between the measured and modeled FWHM (see Figure S2, Supporting Information, and Walsh et al. ${ }^{[1]}$ for further details of PSF fitting). The PSF was dependent on many factors including but not limited to the wavelength of the fluorophore, the concentration of the opacifying agent, and pixel size (zoom level) of the microscope. Final PSF parameter values for each image stack with imaging parameters are shown in Table S1, Supporting Information. In the final stage, the PSF was made asymmetric (due to physical sectioning discussed) by setting the lower half to zero and subtracting the background in the upper half (pixel intensities less than 0.0001 in 32 bit images were zeroed). This PSF was then used in a GPU-accelerated RL deconvolution algorithm ${ }^{[35]}$ with border size of one-fourth of the image stack in each dimension, respectively. Optimization of iterations was based on orthogonality of structures and contrast-to-noise ratio in the deconvolved final image stack. ${ }^{[1]}$

Murine Tumor Xenograft Model: About 8-10-week-old, female, immune-compromised nu/nu nude mice (background CD1) were used (Charles River Laboratories). Cells from the FaDu cell line (a hypopharyngeal cancer cell line gifted by Dr. Craig Murdoch [Sheffield University]) were cultured in complete medium (Dulbecco's minimum essential medium Eagle with L-glutamine [DMEM] [Lonza] $+10 \%$ fetal bovine serum [Invitrogen]) in the ratio $1: 10$ ( $\mathrm{vol} / \mathrm{vol})$ and incubated at $37^{\circ} \mathrm{C}$ and $5 \%$ $\mathrm{CO}_{2}$. To prepare for injection, cells were washed with Dulbecco's PBS and detached with trypsin-EDTA $\left(7-8 \mathrm{~min}, 37^{\circ} \mathrm{C}, 5 \% \mathrm{CO}_{2}\right)$ (Sigma). Cells were labeled with CMDil (Thermofisher UK), a medium-term fluorescent cell-tracking dye that endures for $\approx 3-6$ cell divisions, and was transferred through cell division (but not cell-cell contact). Stain was dissolved from stock concentration ( $1 \mathrm{mg} \mathrm{mL}^{-1}$ in ethanol) in D-PBS to a working solution of $1 \mu \mathrm{M}$. Cells were incubated in the working solution for $5 \mathrm{~min}$ at $37^{\circ} \mathrm{C}$ and then for $15 \mathrm{~min}$ at $4{ }^{\circ} \mathrm{C}$. Cells were then washed and resuspended in PBS for injection. A $100 \mu$ bolus of $1 \times 10^{6}$ cells 
was injected subcutaneously into the left flank above the hind leg of each mouse $(N=5)$ and unstained cells were injected into the right flank also in a $100 \mu$ bolus of $1 \times 10^{6}$ cells. Tumor growth was measured daily with calipers every day after the tumor became palpable and were grown until total tumor volume was $1500 \mathrm{~mm}^{3}$ or 3 weeks postinjection had elapsed.

For blood vessel staining, $200 \mu$ lectin ("Tomato") bound to DyeLyte649 (Vector UK) (1 $\left.\mathrm{mg} \mathrm{mL}^{-1}\right)$ was administered via tail vein injection and allowed to circulate for $10 \mathrm{~min}$ before perfusion fixation to allow sufficient binding to the vascular endothelium. ${ }^{[3]}$

Applications Segmentation: For each application, tumor, kidney, and brain, an example segmentation for the biological structure of interests was conducted to demonstrate the quantification potential of MFHREM data.

Glomeruli segmentation was conducted in Vaa3D (v3.601) using the gradient vector flow algorithm, ${ }^{[57-59]}$ with diffusion iteration of 5 . This algorithm is a widely used extension to a traditional active contour segmentation technique, where the external energy term in the traditional active contour algorithm is replaced with the gradient vector flow field. Manual segmentation of the kidney cortex was then conducted to remove structures not within the cortex (i.e., not glomeruli). Finally, a connected component analysis was conducted in Amira v 19.2 with a threshold max size of $20000 \mu^{3}$. No deconvolution was necessary (as measured by orthogonality ratio) due to the sizes of the structures of interest (glomeruli) relative to the pixel size.

For the tumor, following deconvolution (parameters in Table S1, Supporting Information), vascular segmentation was conducted via the APP2 algorithm of Vaa3D (v3.601). ${ }^{[57-60]}$ Parameters were set as follows: threshold $=-1$ (autothresholding), $\mathrm{CNN}=3$, and Grey scale distance transform (GSDT) and other parameters were used at their default values.

Brain microvasculature segmentation was conducted after deconvolution, semimanually in Amira-Avizo V 2019.2 using a 3D magic wand tool. White-matter orientation analysis was conducted using the Orientation plugin of Fiji ${ }^{[4-47]}$ with a Gaussian gradient and kernel sizes of eight pixels or four pixel for Figure 7D,F, respectively.

HREM Imaging: All MF-HREM imaging was conducted using the Optical-HREM instrument (Indigo Scientific, UK), as shown in Figure 1. Samples were prepared into blocks, as shown in Table 1. Blocks were then mounted into the instrument and trimmed until a flat-block surface perpendicular to the optical axis was created. Once this surface was achieved, the desired slice thickness, $x, y$ resolution, focus, total imaging depth, and exposure and gain for each wavelength were set. An air blower and vacuum were positioned to remove serial slices after sectioning. Imaging then proceeded in a fully automated manner until the total imaging depth was achieved. For each application, imaging parameters are shown in Table S1, Supporting Information.

\section{Supporting Information}

Supporting Information is available from the Wiley Online Library or from the author.

\section{Acknowledgements}

The authors would like to thank Craig Murdoch for the kind gift of the FaDu cell line.

\section{Conflict of Interest}

The authors declare no conflict of interest.

\section{Data Availability Statement}

The data that support the findings of this study are openly available in Image Data Resource (IDR) at https://doi.org/10.17867/10000143. ${ }^{[62]}$

\section{Keywords}

deconvolution, high-resolution episcopic microscopy, serial-sectioning, tumors, whole mounts

Received: May 11, 2021

Published online:

[1] N. Renier, Z. Wu, D. J. Simon, J. Yang, P. Ariel, M. Tessier-Lavigne, Cell 2014, 159, 896.

[2] F. F. Voigt, D. Kirschenbaum, E. Platonova, S. Pagès, R. A. A. Campbell, R. Kastli, M. Schaettin, L. Egolf, A. van der Bourg, P. Bethge, K. Haenraets, N. Frézel, T. Topilko, P. Perin, D. Hillier, S. Hildebrand, A. Schueth, A. Roebroeck, B. Roska, E. T. Stoeckli, R. Pizzala, N. Renier, H. U. Zeilhofer, T. Karayannis, U. Ziegler, L. Batti, A. Holtmaat, C. Lüscher, A. Aguzzi, F. Helmchen, Nat. Methods 2019, 16, 1105.

[3] A. d'Esposito, P. W. Sweeney, M. Ali, M. Saleh, R. Ramasawmy, T. A. Roberts, G. Agliardi, A. Desjardins, M. F. Lythgoe, R. B. Pedley, R. Shipley, S. Walker-Samuel, Nat. Biomed. Eng. 2018, 2, 773.

[4] T. Liebmann, N. Renier, K. Bettayeb, P. Greengard, M. TessierLavigne, M. Flajolet, Cell Rep. 2016, 16, 1138.

[5] J. A. Gleave, J. P. Lerch, R. M. Henkelman, B. J. Nieman, PLoS One 2013, 8, e72039.

[6] K. Tainaka, T. C. Murakami, E. A. Susaki, C. Shimizu, R. Saito, K. Takahashi, A. Hayashi-Takagi, H. Sekiya, Y. Arima, S. Nojima, Cell Rep. 2018, 24, 2196.

[7] J. H. Kim, M. J. Jang, J. Choi, E. Lee, K. D. Song, J. Cho, K. T. Kim, H. J. Cha, W. Sun, Sci. Rep. 2018, 8, 1.

[8] T. Yu, Y. Qi, H. Gong, Q. Luo, D. Zhu, J. Biophotonics 2018, 11, e201700187.

[9] D. G. C. Hildebrand, M. Cicconet, R. M. Torres, W. Choi, T. M. Quan, J. Moon, A. W. Wetzel, A. S. Champion, B. J. Graham, O. Randlett, Nature 2017, 545, 345.

[10] G. Krishnamurthi, C. Y. Wang, G. Steyer, D. L. Wilson, Opt. Express 2010, 18, 22324.

[11] a) C. Walsh, N. Holroyd, R. Shipley, S. Walker-Samuel, in Proc.Medical Image Unerstanding and Analysis, (Eds: B. W. Papież, A. I. L. Namburete, M. Yaqub, J. A. Noble), Springer International Publishing 2020, p. 235; b) C. Walsh, presented at 24th Annual Conf., Oxford, July 2020.

[12] S. P. Amato, F. Pan, J. Schwartz, T. M. Ragan, Front. Neuroanat. 2016, 10,1

[13] K. Seiriki, A. Kasai, T. Nakazawa, M. Niu, Y. Naka, M. Tanuma, H. Igarashi, K. Yamaura, A. Hayata-Takano, Y. Ago, H. Hashimoto, Nat. Protoc. 2019, 14, 1509.

[14] H. Gong, D. Xu, J. Yuan, X. Li, C. Guo, J. Peng, Y. Li, L. A. Schwarz, A. Li, B. Hu, B. Xiong, Q. Sun, Y. Zhang, J. Liu, Q. Zhong, T. Xu, S. Zeng, Q. Luo, Nat. Commun. 2016, 7, 1.

[15] L. Abdeladim, K. S. Matho, S. Clavreul, P. Mahou, J.-M. Sintes, I. Solinas, X. Arganda-Carreras, S. G. Turney, J. W. Lichtman, A. Chessel, K. Bemelmans, A.-P. Loulier, W. Supatto, J. Livet, E. Beaurepaire, Nat Commun. 2019, 10, 1662.

[16] S. H. Geyer, B. Maurer-Gesek, L. F. Reissig, W. J. Weninger, J. Vis. Exp. 2017, 125, e56071.

[17] W. J. Weninger, S. H. Geyer, A. Martineau, A. Galli, D. J. Adams, DMM 2014, 7, 1143.

[18] G. Pieles, S. H. Geyer, D. Szumska, J. Schneider, S. Neubauer K. Clarke, K. Dorfmeister, A. Franklyn, S. D. Brown, S. Bhattacharya, W. J. Weninger, J. Anat. 2007, 211, 132.

[19] W. J. Weninger, S. H. Geyer, T. J. Mohun, D. Rasskin-Gutman, T. Matsui, I. Ribeiro, L. da F. Costa, J. C. Izpisua-Belmonte, G. B. Muller, Anat. Embryol. 2006, 211, 213. 
[20] T. Mohun, D. J. Adams, R. Baldock, S. Bhattacharya, A. J. Copp, M. Hemberger, C. Houart, M. E. Hurles, E. Robertson, J. C. Smith, T. Weaver, W. J. Weninger, DMM 2013, 6, 562.

[21] J. Rosenthal, V. Mangal, D. Walker, M. Bennett, T. J. Mohun, C. W. Lo, Birth Defects Res. C Embryo Today 2004, 72, 213.

[22] T. J. Mohun, W. J. Weninger, Curr. Opin. Genet. Dev. 2011, 21, 573.

[23] S. H. Geyer, M. M. Nöhammer, I. E. Tinhofer, W. J. Weninger, J. Anat. 2013, 223, 603.

[24] H. Matsui, S. Y. Ho, T. J. Mohun, H. M. Gardiner, Ultrasound Obstet. Gynecol. 2015, 45, 492.

[25] W. J. Weninger, T. J. Mohun, Methods Mol. Biol. 2007, 411, 35.

[26] T. J. Mohun, W. J. Weninger, Cold Spring Harb. Protoc. 2012, 7, 678.

[27] D. J. Jafree, D. Moulding, M. Kolatsi-Joannou, N. P. Tejedor, K. L. Price, N. J. Milmoe, C. L. Walsh, R. M. Correra, P. J. D. Winyard, P. C. Harris, C. Ruhrberg, S. Walker-Samuel, P. R. Riley, A. S. Woolf, P. J. Scambler, D. A. Long, Elife 2019, 8, e48183.

[28] W. Li, R. N. Germain, M. Y. Gerner, Proc. Natl. Acad. Sci. 2017, 114, e7321.

[29] E. Chee Tak Yeung, Plant Microtechniques and Protocols, Springer, Cham 2015.

[30] Z. Yang, B. Hu, Y. Zhang, Q. Luo, H. Gong, PLoS One 2013, 8, 4.

[31] Y. Gang, X. Liu, X. Wang, Q. Zhang, H. Zhou, R. Chen, L. Liu, Y. Jia, F. Yin, G. Rao, J. Chen, S. Zeng, Biomed. Opt. Express 2017, 8, 3583.

[32] A. J. Ewald, H. Mcbride, M. Reddington, S. E. Fraser, R. Kerschmann, Dev. Dyn. 2002, 225, 369.

[33] W. Wallace, L. H. Schaefer, J. R. Swedlow, BioTechniques 2001, 31, 1076.

[34] F. Soulez, L. Denis, Y. Tourneur, É. Thiébaut, in 9th IEEE Int. Symp. on Biomedical Imaging (ISBI), 2012, 1735.

[35] C. Rueden, C. Dietz, M. Horn, J. Schindelin, B. Northan, M. Berthold, K. Eliceiri, Image) Ops [software], 2016.

[36] E. J. Baldelomar, J. R. Charlton, S. C. Beeman, B. D. Hann, L. CullenMcEwen, V. M. Pearl, J. F. Bertram, T. Wu, M. Zhang, K. M. Bennett, Kidney Int. 2016, 89, 498.

[37] A. Klingberg, A. Hasenberg, I. Ludwig-Portugall, A. Medyukhina, L. Männ, A. Brenzel, D. R. Engel, M. T. Figge, C. Kurts, M. Gunzer, J. Am. Soc. Nephrol. 2017, 28, 452.

[38] D. Hanahan, R. A. Weinberg, Cell 2011, 144, 646.

[39] P. Friedl, K. Wolf, Nat. Rev. Cancer 2003, 3, 362.

[40] T. Magdeldin, V. López-Dávila, J. Pape, G. W. W. Cameron, M. Emberton, M. Loizidou, U. Cheema, Sci. Rep. 2017, 7, 1.

[41] X. Zhang, X. Yin, J. Zhang, A. Li, H. Gong, Q. Luo, H. Zhang, Z. Gao, H. Jiang, Natl. Sci. Rev. 2019, 6, 1223.

[42] J. Steinman, M. M. Koletar, B. Stefanovic, J. G. Sled, PLoS One 2017, 12, e0186676.

[43] M. I. Todorov, J. C. Paetzold, O. Schoppe, G. Tetteh, S. Shit, V. Efremov, K. Todorov-Völgyi, M. Düring, M. Dichgans, M. Piraud, B. Menze, A. Ertürk, Nat. Methods 2020, 17, 442.
[44] M. D. Budde, J. A. Frank, Neuroimage 2012, 63, 1.

[45] Z. Püspöki, M. Storath, D. Sage, M. Unser, Focus on Bio-Image Informatics, Springer, Cham 2016, p. 69.

[46] R. Rezakhaniha, A. Agianniotis, J. T. C. Schrauwen, A. Griffa, D. Sage, C. V. C. vd Bouten, F. N. Van De Vosse, M. Unser, N. Stergiopulos, Biomech. Model. Mechanobiol. 2012, 11, 461.

[47] E. Fonck, G. G. Feigl, J. Fasel, D. Sage, M. Unser, D. A. Rüfenacht, N. Stergiopulos, Stroke 2009, 40, 2552.

[48] R. Cai, C. Pan, A. Ghasemigharagoz, M. I. Todorov, B. Förstera, S. Zhao, H. S. Bhatia, A. Parra-Damas, L. Mrowka, D. Theodorou, M. Rempfler, A. L. R. Xavier, B. T. Kress, C. Benakis, H. Steinke, S. Liebscher, I. Bechmann, A. Liesz, B. Menze, M. Kerschensteiner, M. Nedergaard, A. Ertürk, Nat. Neurosci. 2019, 22, 317.

[49] J. Kohl, J. Ng, S. Cachero, E. Ciabatti, M.-J. Dolan, B. Sutcliffe, A. Tozer, S. Ruehle, D. Krueger, S. Frechter, T. Branco, M. Tripodi, G. S. X. E. Jefferis, Proc. Natl. Acad. Sci. 2014, 111, e3805.

[50] Y. Qi, T. Yu, J. Xu, P. Wan, Y. Ma, J. Zhu, Y. Li, H. Gong, Q. Luo, D. Zhu, Sci. Adv. 2019, 5, eaau8355.

[51] J. Guo, C. Artur, J. L. Eriksen, D. Mayerich, Sci. Rep. 2019, 9, 14578.

[52] D. K. Samuylov, P. Purwar, G. Szekely, G. Paul, IEEE Trans. Image Process. 2019, 28, 3688.

[53] J. E. D. Zamboni, V. H. Casco, J. Imaging 2017, 3.

[54] R. J. Vigouroux, M. Belle, A. Chédotal, Mol. Brain 2017, 10, 1.

[55] P. Workman, E. O. Aboagye, F. Balkwill, A. Balmain, G. Bruder, D. J. Chaplin, J. A. Double, J. Everitt, D. A. H. Farningham, M. J. Glennie, L. R. Kelland, V. Robinson, I. J. Stratford, G. M. Tozer, S. Watson, S. R. Wedge, S. A. Eccles, Br. J. Cancer 2010, $102,1555$.

[56] J. Schindelin, I. Arganda-Carreras, E. Frise, V. Kaynig, M. Longair, T. Pietzsch, S. Preibisch, C. Rueden, S. Saalfeld, B. Schmid, J.-Y. Tinevez, D. J. White, V. Hartenstein, K. Eliceiri, P. Tomancak, A. Cardona, Nat. Methods 2012, 9, 676.

[57] H. Peng, A. Bria, Z. Zhou, G. Iannello, F. Long, Nat. Protoc. 2014, 9, 193.

[58] H. Peng, Z. Ruan, F. Long, J. H. Simpson, E. W. Myers, Nat. Biotechnol. 2010, 28, 348.

[59] H. Peng, J. Tang, H. Xiao, A. Bria, J. Zhou, V. Butler, Z. Zhou, P. T. Gonzalez-Bellido, S. W. Oh, J. Chen, A. Mitra, R. W. Tsien, H. Zeng, G. A. Ascoli, G. lannello, M. Hawrylycz, E. Myers, F. Long, Nat. Commun. 2014, 5, 4342.

[60] H. Xiao, H. Peng, Bioinformatics 2013, 29, 1448.

[61] D. Sage, L. Donati, F. Soulez, D. Fortun, G. Schmit, A. Seitz, R. Guiet, C. Vonesch, M. Unser, Methods 2017, 115, 28.

[62] C. Walsh, N. Holroyd, E. Finnerty, S. G. Ryan, P. W. Sweeney, R. J. Shipley, S. Walker-Samuel, idr0085-walsh-mfhrem/experimentA, https://doi.org/10.17867/10000143. 